\title{
DESIGN OF THREE-CHANNEL OPTICAL RECEIVING SYSTEM FOR DUAL- FREQUENCY LASER RADAR
}

\author{
Jiandong Wei ${ }^{1,2}$, Guoqing Zhou ${ }^{1,2,3}$ Xiang Zhou ${ }^{1,2,3 *}$, JinlongChen ${ }^{1,2}$, YizhiTan ${ }^{1,2}$,HaochengHu ${ }^{1,2}$ \\ ${ }^{1}$ Department of Mechanical and Control Engineering, Guilin University of Technology, No. 12 Jian'gan Road, Guilin, \\ Guangxi541004, China-961466473@qq.com \\ ${ }^{2}$ Guangxi Key Laboratory of Spatial Information and Geomatics, Guilin University of Technology, No. 12 Jian'gan Road, Guilin, \\ Guangxi 541004, China - (gzhou, zqx0711)@glut.edu.cn \\ ${ }^{3}$ School of Microelectronics, Tianjin University, No. 92 Weijin Road, Tianjin 300072, China - (gzhou, zqx0711)@glut.edu.cn
}

\section{Commission III, WG III/1}

KEY WORDS: Radar, Coastal, Optical receiving system, Dual frequency, Three channels

\begin{abstract}
:
There are many intertidal zones and island reefs in the coastal area, the traditional shipborne acoustic measurement method is extremely inefficient, so the coastal area three-dimensional integrated measurement has always been a difficult point in the field of remote sensing. Because the sea blue-green light window has good transparency and the laser point cloud data can quickly and accurately distinguish the characteristics of shallow sea water topography. At present, the most effective detection method for coastal areas in the world is the airborne dual-frequency laser radar detection technology, which has high measurement rate and wide coverage. The laser outputs both $1064 \mathrm{~nm}$ and $532 \mathrm{~nm}$ dual-wavelength laser, $1064 \mathrm{~nm}$ laser forms sea surface echo, and 532nm laser penetrates sea water to form shallow sea and deep sea echo. However, during the propagation of seawater, the number of photon scattering increases with the increase of water depth, which will cause the attenuation of the echo signal. As a result, the detection of weak light in the large dynamic range is not high, which has been a difficult point for near-shore airborne sounding. To solve this problem, we designed a split-field, three-channel optical receiving system. The ZEMAX simulation results show that the dual-channel laser radar three-channel receiving optical system effectively reduces the optical crosstalk between the optical components and the channels, and achieves energy harvesting in different water depth channels. The structure dynamically compresses the photoelectric signal and improves the signal to noise ratio.
\end{abstract}

\footnotetext{
* Corresponding author: Xiang Zhou; Email: zqx0711@glut.edu.cn
} 


\section{INSTRUCTIONS}

The coast has many intertidal zones and island reefs. The traditional detection method uses multi-beam detection technology to achieve shallow sea mapping by surface scanning, but in the actual application process, the blind zone has many dead angles and low efficiency (Yang 2011(1)). Therefore, the threedimensional comprehensive measurement of coastal zones has always been a difficult point in the field of remote sensing, and it is impossible to effectively survey underwater objects and underwater topography from the air. The application of airborne laser scanning ranging technology in underwater detection is mainly due to the diversified development of single-frequency airborne laser radar technology (Qi 2011). Lidar scanning technology will combine a variety of image data, multi-spectral data to obtain more scanning information features, enabling airborne laser point cloud data to more accurately and conveniently achieve interpretation of terrain features, spatial information Measurement and acquisition (). The seawater has a transmission window in the blue-green light band, and the green light at $532 \mathrm{~nm}$ in the window has strong seawater penetration ability. The airborne dual-frequency lidar detection technology uses this transmission window to emit blue-green band lasers and $1064 \mathrm{~nm}$ infrared light from the aerial platform to the seawater, and measures the depth of the seawater according to the time difference of the laser reflection on the sea surface and the sea floor(Guo 2013) .The use of high repetitive frequency lasers to extract dynamic large-scale weak optical signals, to achieve the identification of weak optical signals, but can not meet the accuracy of water and land data acquisition.

Our research team won a special focus on innovation and development in Guangxi in 2018. The purpose of this project is to develop an airborne laser high precision 3D seabed instrument (LiDAR). The principle structure diagram of the instrument is shown in Figure 1.This paper combines airborne blue-green and infrared dual-frequency laser scanning technology to construct an airborne dual-frequency laser radar technology, which can not only detect underwater targets, but also achieve rapid joint measurement of water and land. The airborne dual-frequency laser radar uses a mature Nd:YAG laser. The 532nm laser that penetrates the seawater is multiplied by the $1064 \mathrm{~nm}$ fundamental of the laser. Therefore, the laser can simultaneously output $1064 \mathrm{~nm}$ and $532 \mathrm{~nm}$ dual-wavelength lasers to form a sea-land integration. The laser outputs both $1064 \mathrm{~nm}$ and $532 \mathrm{~nm}$ dualwavelength laser, $1064 \mathrm{~nm}$ laser forms sea surface echo, and $532 \mathrm{~nm}$ laser penetrates sea water to form shallow sea and deep sea echo. However, during the propagation of seawater, the number of photon scattering increases with the increase of water depth, which will cause the attenuation of the echo signal. As a result, the detection of weak light in the large dynamic range is not high, which has been a difficult point for near-shore airborne sounding. To solve this problem, we designed a split-field, three-channel optical receiving system. The receiving optical system is designed with three echo receiving channels: sea surface, shallow water and deep water. Each receiving channel corresponds to different detector modules.

\section{AIRBORNE LIDAR SEAWATER SOUNDING PRINCIPLE}

Since the sea surface emits strong background light and affects the detection sensitivity, the near-infrared spectrum is selected as the laser signal for detecting the sea surface, and is removed by the narrow band filter. The influence of the solar background light is initially based on the $1064 \mathrm{~nm}$ spectrum. The optimal spectral transmission window of the coastal waters is $520 \mathrm{~nm}$. Considering the actual width of the seawater window and the $532 \mathrm{~nm}$ green laser spectrum used in the industry, the green emission spectrum is $532 \mathrm{~nm}$ for the laser emission signal of the seabed signal. The launch system emits two different wavelengths of laser pulses to the sea surface - $1064 \mathrm{~nm}$ infrared light and $532 \mathrm{~nm}$ green light. Since infrared light does not easily penetrate seawater, most of it is reflected when it encounters seawater. Used to measure the height of the aircraft and the sea surface. The $532 \mathrm{~nm}$ green light is located right in the "wave band of the ocean blue-green optical window". The laser can be reflected back through the sea water until the bottom of the sea to detect the bottom of the water[5]. The water depth at a point can be calculated from the time interval between the water surface and the bottom of the two laser echoes. The radar system can acquire the three-dimensional topography of the surface and the seabed through the positioning and attitude information of the platform.

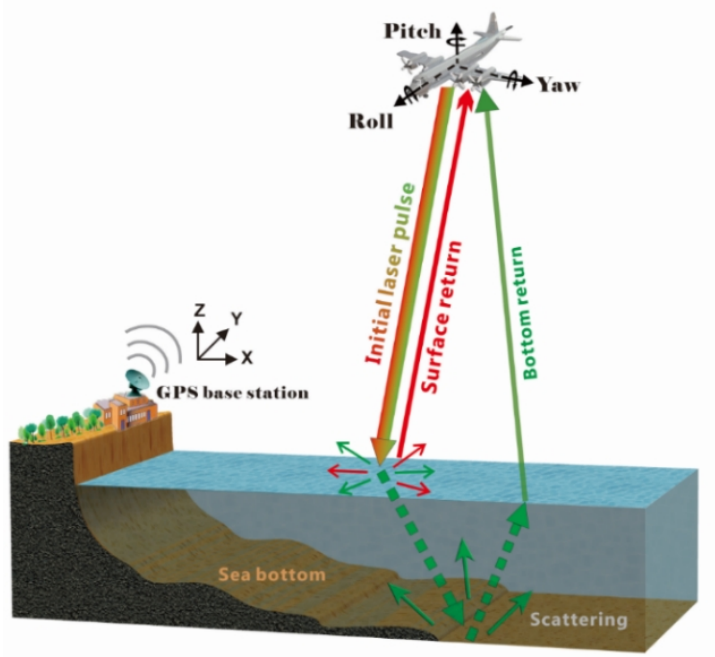

Figure 1. Dual LIDAR radar working diagram

In the dual-frequency laser radar system, the lidar equation can be used to describe the relationship between the laser action distance and the echo signal strength, and the diameter of the target, the distance of the action, the optical parameters of the system, the attenuation of the medium, etc. The relationship between the laser radar target scattering signal can be established. In order to simplify the operation, the target is assumed to be the Lambertian surface, and the power of the laser beam is evenly distributed over the target[8].

$$
\mathrm{P}_{r}=\rho T_{\mathrm{a}}^{2} \eta_{\mathrm{t}} \eta_{\mathrm{r}} \cos \theta_{\mathrm{t}} \frac{A_{\mathrm{t}}}{A_{l}} \cdot \frac{A_{r}}{\pi R^{2}} \mathrm{P}_{\mathrm{t}}
$$

where $\rho=$ diffuse reflection target reflectance of the laser

$T_{a}=$ single pass transmission rate of the working medium between the target and the system.

$\eta_{\mathrm{t}}=$ optical efficiency of the laser emission system.

$\theta_{\mathrm{t}}=$ normal angle between the optical axis of the laser emitting system and the target.

$A_{\mathrm{t}}=$ projected area of the irradiated portion of the laser emitting beam in the field of view.

$A_{l}=$ cross-sectional area of the laser beam in the field of view of the lidar at the target.

$A_{r}=$ effective receiving area of the receiving optical system. 


$$
\begin{aligned}
& R=\text { distance between the target and the lidar } \\
& \mathrm{P}_{\mathrm{t}}=\mathrm{t} \text { power of the laser. }
\end{aligned}
$$

For a planar target, when the area of the target is large, the projected portion of the target in the field of view has a large projection area in the cross section of the laser emitting beam, and all laser energy scattered at the spot is received by the laser receiving system, and then the laser is received once. During the process of detection, $A_{\mathrm{t}}$ and $A_{l}$ are considered to be approximately equal. The dual-frequency laser radar system is a lattice spiral scan, and the laser beam cross-sectional area in the laser radar field of view at the target is also the spot size is exactly equal to the target illuminated portion of the field of view. The formula can be simplified as follows:

$$
\mathrm{P}_{r}=\rho T_{\mathrm{a}}^{2} \eta_{\mathrm{t}} \eta_{\mathrm{r}} \cos \theta_{\mathrm{t}} \cdot \frac{A_{r}}{\pi R^{2}} \mathrm{P}_{\mathrm{t}}
$$

The sea surface echo energy is strong, while the seafloor echo is weak due to attenuation and backscattering. The dual-frequency laser LiDAR transceiver component has a large dynamic range.

According to the radar equation, according to the total input laser emission pulse energy of $3 \mathrm{~mJ}$, the solar background light is suppressed by the filter of about $1 \mathrm{~nm}$, the sea surface echo power and the seafloor echo power are as follows:

\begin{tabular}{|l|l|l|}
\hline laser energy & $3.5 \mathrm{~mJ} @ 1064 \mathrm{~nm} \& 532 \mathrm{~nm}$ \\
\hline filter bandwidth & $1.2 \mathrm{~nm}$ \\
\hline filter transmittance & 0.88 \\
\hline $\begin{array}{l}\text { system transmittance } \\
\text { and caliber }\end{array}$ & $0.72 \% 、 100 \mathrm{~mm}$ \\
\hline $\begin{array}{l}\text { solar background } \\
\text { power }\end{array}$ & $1064 \mathrm{~nm}$ & $3.2 \times 10-8 \mathrm{~W}$ \\
\cline { 2 - 3 } $\begin{array}{l}\text { sea surface echo } \\
\text { power (reflected by } \\
1.4 \% \text { of the sea } \\
\text { surface) }\end{array}$ & $532 \mathrm{~nm}$ & $3.9 \times 10-8 \mathrm{~W}$ \\
\hline \multirow{2}{*}{\begin{tabular}{l} 
submarine echo power \\
\cline { 2 - 3 }
\end{tabular}} & $\begin{array}{l}532 \mathrm{~nm} \\
\text { quality }\end{array}$ & $2.7 \times 10-4 \mathrm{~W}$ \\
\hline & Bedium & $1.2 \times 10-9 \mathrm{~W}$ \\
\hline
\end{tabular}

Table 1 Calculation results of solar background power and echo power of different conditions

Among them, because different water depths and water quality have different attenuation of laser energy(Zhou 2015), when the depth of detection is $50 \mathrm{~m}$, the calculation of seafloor echo energy is divided into better water quality conditions (seawater diffusion attenuation coefficient is $0.08 \mathrm{~m}^{-1}$ ) with medium water quality (seawater flooding). The attenuation coefficient is $0.12 \mathrm{~m}^{-}$ $\left.{ }^{1}\right)$.

\section{DUAL-FREQUENCY LIDAR SYSTEM RECEIVING FIELD OF VIEW SEGMENTATION}

The field of view is the range of areas that can be observed by the imaging system, that is, the range of objects seen from the image plane. At the infinity, the detector is a field diaphragm, placed on the focal plane of the objective lens, and coincides with the window. The field of view of the optical receiving system represents the range that the detector can detect in the object space through the optical receiving system. The size of the field of view is usually represented by the plane field of view.

The attenuation of the laser during seawater transmission has a great relationship with the FOV of the receiver. When the photon is scattered multiple times, the final exit direction exceeds the receiving FOV range, which will cause photon loss. Therefore, in order to enable the laser to receive a deeper range of echo signals and increase the laser sounding capability, the receiver FOV is generally large, assuming that the FOV is large enough to cover the cross section of the laser divergence angle on the water surface, the signal caused by the FOV The loss will not have a serious impact on the laser pulse echo signal in shallow water measurements[5]. As the water depth increases, the number of photon scattering increases, and the more photons that eventually exceed the range of the received field of view, the greater the effect on the laser pulse echo signal. At the same time, it is also considered that the larger the receiving field of view, the greater the background solar noise entering the receiver, which in turn reduces the signal-to-noise ratio of the echo signal. Therefore, dividing the different field of view according to the depth of detection is especially important for the accuracy of the entire receiving system.


field distribution

Figure 2. Field of view segmentation

Consider the dual-frequency lidar system scanning grid point density of $2 \mathrm{~m} \times 2 \mathrm{~m}$, flying height of $1000 \mathrm{~m}$. The diameter of the spot formed by the sea surface after $1064 \mathrm{~nm}$ laser should be less than $2 \mathrm{~m}$. In order to avoid the specular reflection of the sea surface and the influence of the peaks and valleys of the wave on the depth of detection, the spot diameter should not be too small. Considering the appeal factor, the laser divergence angle is initially set at $1 \mathrm{mrad}$. Due to the small scattering effect of light in the atmospheric medium, the spot size of the laser on the sea surface is about $1 \mathrm{~m}$ at a flying height of $1 \mathrm{~km}$. At the same time, the scanning direction is about $10^{\circ}$ from the lower point of the machine, and the influence on the instantaneous field of view can be ignored. For the sea surface channel, the receiving FOV can cover the emission spot, considering the redundancy to take $2 \mathrm{mrad}$. In order to ensure more energy in the deep water channel, the field of view of the shallow water channel can be sacrificed, 
and the field of view of the shallow water channel is $5 \mathrm{mrad}$. Considering the requirements for miniaturization and light weight of the product, the field of view of the Cassegrain optical system cannot be made too large, and the receiving field of the deep water channel takes $50 \mathrm{mrad}$.

\section{STRUCTURAL DESIGN OF DUAL-CHANNEL LIDAR THREE-CHANNEL RECEIVING SYSTEM}

The receiving optics system is designed with three echo receiving channels: sea surface, shallow water and deep water. Each receive channel corresponds to a different detector module. The echo signals from the sea surface and the sea floor are received by the Cassegrain telescope system, which uses a large aperture receiving objective to increase the field of view (FOV) of the receiving system. Three echoes into the receiving system, including $1064 \mathrm{~nm}$ sea surface echoes, and $532 \mathrm{~nm}$ shallow sea echoes and $532 \mathrm{~nm}$ deep water echoes. The device adopts a method of splitting the beam and then dividing the field of view, and the $1064 \mathrm{~nm}$ echo is reflected by the dichroic beam splitter into the sea channel. The $1064 \mathrm{~nm}$ echo is collimated in the channel and incident on the photosensitive surface of the APD. Because the cross section of the light in the seawater is close to half the depth of the water. Through the $532 \mathrm{~nm}$ echo of the dichroic beam splitter, the subfield of view is used to separately collect echoes of different depths. On the horizontal light path after the $532 \mathrm{~nm}$ echo passes through the dichroic mirror. For a central small field of view of $5 \mathrm{mrad}$, a $45^{\circ}$ mirror is placed and embedded in the center of the first mirror in the collimated structure of the large field of view (Zhou. G). The $532 \mathrm{~nm}$ echo of the small field of view is reflected into the shallow sea channel and is in the single quadrant PMT. The remaining 5 50mrand edge large field of view acts as a deepwater channel, and the $532 \mathrm{~nm}$ echo of the collimated large field of view is projected onto the high gain four quadrant PMT detector module.

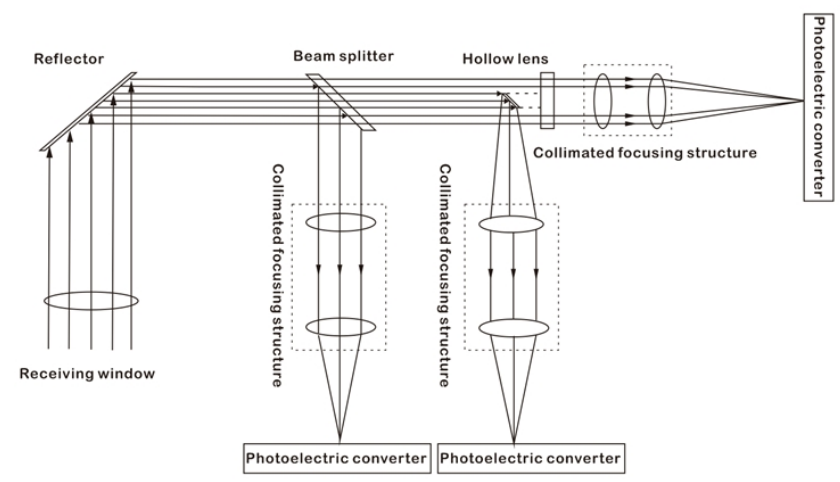

Figure 3. Three-channel structure diagram

As shown in Figure 3, the innovative design of a new lens structure. A mirror is embedded in the middle of the hollow lens and extends in a columnar shape. Then install a mirror on the $45^{\circ}$ slope to achieve the purpose of splitting the field of view. Because the cross section of the light in the seawater is close to half the depth of the water. The amount of photon scattering increases as the water depth increases. Therefore, the deep water echo is received in a large field of view, and the shallow water echo is received in a small field of view. In order to avoid the small field of view to form a occlusion of the large field of view. Therefore, the center of the hollow lens is extended to embed a mirror of $45^{\circ}$.

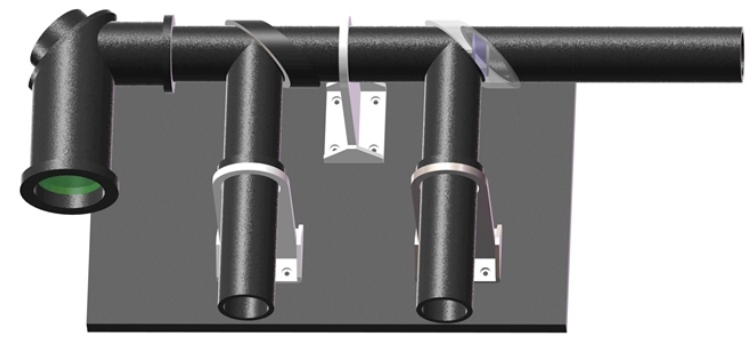

Figure 4. 3D model simulation

\section{EXPERIMENT ANALYSIS}

The ZEMAX software simulates optical system models such as reflection, refraction, diffraction, spectroscopic, and coating, and analyzes various aberrations $\mathrm{Xu} 2013)$. When simulating the telescope port system, the entire optical path is divided into three parts according to the above structure. The first reflected light path corresponds to the $532 \mathrm{~nm}$ deep sea channel, and the other two light paths correspond to the $532 \mathrm{~nm}$ shallow sea channel and the $1064 \mathrm{~nm}$ sea surface pass channel respectively. The difference in position of the two laser emission windows causes the spot position to be different.

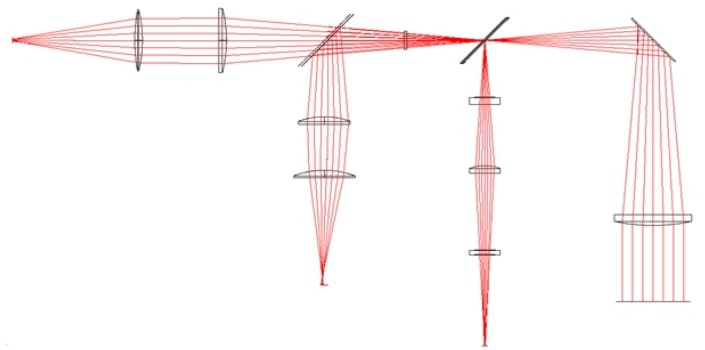

Figure 5. Three-channel optical receiving system simulation

The total optical field of view of the receiving optical system is $50 \mathrm{mrad}$, and the small field of view of $5 \mathrm{mrad}$ in the middle is taken as a shallow water channel, while the remaining field of view of 5 to $50 \mathrm{mrad}$ is a deep water channel. Since the echo signal has a weak background light, it is proposed to use the relevant processing means for detection. Therefore, a high-gain fourquadrant PMT is selected as the detector module.

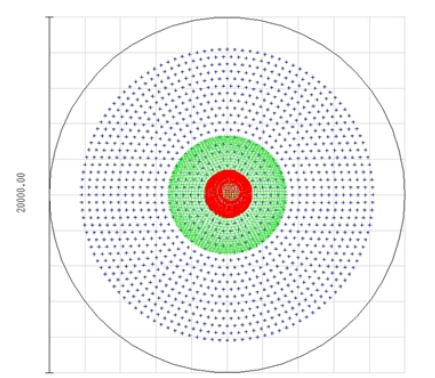

Figure 6. Field segmentation point map

\section{CONCLUSIONS}

The field of view is segmented according to the detection target and the depth of the detection, and then the different channels are received by the sub-channels, and further collimated in each channel, so that each echo reaches the corresponding photodetector when the energy reaches the maximum. Through this sub-channel, the optical crosstalk between the optical element and the channel is effectively reduced, and energy collection of different water depth channels is realized. The structure 
dynamically compresses the photoelectric signal and improves the signal-to-noise ratio. By splitting the beam and then dividing the field of view, the filter is avoided in each channel, the axial distance of each channel is reduced, and the size and weight of the device are reduced. It can effectively improve the detection accuracy of airborne dual-frequency laser radar.

\section{ACKNOWLEDGEMENT}

This paper is financially supported by the National Natural Scien ce of China under Grant numbers 41431179,41961065; Guangxi Innovative Development Grand Grant under the grant number: $G$ uikeAA18118038, GuikeAA18242048; the National Key Resear ch and Development Program of China under Grant numbers 20 16YFB0502501 and the BaGuiScholars program of Guangxi

\section{REFERENCES}

Yang Yongchong, $\mathrm{Hu} \mathrm{Nan}$, et al. Research on 3D digital topographic mapping technology[J]. Bulletin of Surveying and Mapping, 2011(1).

Qi Keqiang, Gong Zhihui, Tang Zhiqiang, et al. The principle of airborne LiDAR technology and its application analysis [J], surveying and spatial geographic information. 2011, 34 (1): 144150.

Guo Xiangqian, Hao Weitao, Li Xiang. Research and Prospect Based on Airborne LIDAR Technology [J], Surveying and Spatial Geography Information, 2013, 36(2): 70.

Ruan Guojun Ruan, Huang Yitao, Ouyang Yongzhong, et al. Key technology in the development of airborne laser sounding system [J]. Marine mapping. 2014, 34(3): 73-76.

Zhou. G, Zhou Xiang, Jiazhi Yang, Yue Tao, Xueqin Nong, Oktay Baysal, Flash Lidar Sensor using Fiber Coupled APDs, IEEE Sensor Journal, vol. 15, no. 9, September, 2015, pp. 47584768 .

Zhou. G, Huang Jingjin, and Zhang Guangyun, Evaluation of the wave energy conditions along the coastal waters of Beibu Gulf, China, Energy, vol. 85, pp. 449-457. 2015.

Zhou. G, Jiang. L, Huang. J, Zhang. R, Liu D, Zhou X and Baysal. O, FPGA-based on-board geometric calibration for linear CCD imager, Sensors, 18, 1794; doi:10.3390/s18061794, 2018

Research Progress of Domestic Airborne Dual-Frequency Lidar Detection Technology[J]. Advances in Laser and Optoelectronics, 2018, v.55; No.631(08):7-17.

Xu Mengyuan. Optical performance analysis of water vapor lidar receiving system based on ZEMAX[D]. Ocean University of China, 2013. 